\title{
Prediction of tensile strength of fused deposition modeling (FDM) printed PLA using classic laminate theory
}

\author{
Shilpesh R. Rajpurohit ${ }^{\mathrm{a}}$, Harshit K. Dave ${ }^{\mathrm{a}}$ and Kamlakar P. Rajurkar ${ }^{\mathrm{b}}$
}

${ }^{a}$ Department of Mechanical Engineering, Sardar Vallabhbhai National Institute of Technology, Surat - 395007, India ${ }^{b}$ Department of Mechanical \& Materials Engineering, University of Nebraska - Lincoln, Lincoln - 68588-0454, United States

\begin{tabular}{l}
\hline A R T I C L E I N F O \\
\hline Article history: \\
Received 25 July 2021 \\
Accepted 11 December 2021 \\
Available online \\
11 December 2021 \\
\hline Keywords: \\
Fused deposition Modeling \\
Classic laminate theory \\
Raster angle \\
Layer height \\
Raster width \\
Tensile strength
\end{tabular}

\section{Introduction}

Additive Manufacturing (AM) technique fabricates complex parts layer by layer using computer-aided (CAD) data. An additive manufacturing technique was used to develop industrial prototypes. Recently, AM technique has been significantly enhanced and its applications have been extended to the production of end-use parts. It is necessary to investigate the mechanical properties of the manufactured part to make sure that it meets the mechanical strength requirements for the desired application. The strength and elastic behavior of the AM built parts differ from the raw material due to the layer-by-layer deposition and layer orientation during layer upon layer deposition. The AM produced parts can only be used if their mechanical properties are known and taken into account during the designing of parts depending upon process parameter selection. However, the prediction of the mechanical properties of the 3D printed parts under a real set of working conditions is one of the critical issues that need to be addressed. Hence, it is required to apply an existing appropriate theory that can estimate the mechanical behavior of a printed part (Alaimo et al., 2017; Xia et al., 2019).

Fused Deposition Modeling (FDM) is one of the most widely used AM processes that can produce polymer prototypes and functional end-use parts. In the FDM process, polymer filament is heated to its melting temperature and then extruded through a heated nozzle, depositing material as raster on the heated bed in a layer. After one layer printing, the heated bed moves in a

* Corresponding author

E-mail addresses: harshitkumar@yahoo.com (S. R. Rajpurohit) 
downward direction by a layer thickness to print another layer on the previously deposited layer. Thus, all the FDM printed parts consist of rasters and layers. The adhesion between adjacent rasters and layers depends upon thermal diffusion during the solidification of the semi-solid extruded raster. The bonding strength between the layers and adjacent rasters governs the final strength of FDM printed parts that is dependent on the liquefier dynamics and on melt deposition and solidification. The bonding strength is ruled by printing process parameters and characteristics of the materials used in printing. Generally, it first prints the boundary known as the outer perimeter and after that infill of the layer as either a solid layer or infill pattern in the layer. The infill structure is defined by the filament deposition strategy and build process parameters. Raster width, layer height, air gap, raster angle, and print temperature are some of the critical process parameters that can influence the infill structure (Rajpurohit \& Dave, 2018, 2019; Ameri et al., 2020, 2021; Zhou et al., 2017).

The mechanical properties of composite material can be predicted using the Classic Laminate Theory (CLT). The mechanical properties of the conventional material are homogeneous and isotropic. In contrast, the mechanical properties of the composite material are often heterogeneous and orthotropic. To predict the mechanical properties of composite structure, the CLT is used in which mechanical properties are defined as a function of the stacking sequence and the presence of other material in each layer (Jones, 2014; Kaw, 2005). The FDM fabricated part is identical to the laminated polymer composite, wherein the part is manufactured by the addition of layers or laminates layer-by-layer.

In recent times, several researchers have applied the CLT and analytical model to characterize the mechanical strength of printed parts. Sugavaneswaran and Arumaikkannu (2018) used the CLT to assess the mechanical properties of additively prepared multi-material parts. They also observed higher tensile strength for cross-ply laminates, higher flexural strength for quasi-isotropic laminates and higher shear strength for angle ply laminates. Alaimo et al. (2017) used CLT to derive the mechanical strength of FDM processed parts at various fiber cross-sections. They reported that at $0^{\circ}$ raster deposition, the tensile strength (UTS) decreases with an increase in filament cross-section. On the other hand, at $90^{\circ}$ raster deposition, UTS increased with increment in filament cross-section. Elastic modulus increased with increment in fiber cross-section for both $0^{\circ}$ and $90^{\circ}$ raster deposition.

Casavola et al. (2016) examined the orthotropic mechanical properties as a function of the stacking sequence using CLT for the FDM processed part. The variation in CLT and experimental results was $1.07 \%$ for ABS and 5.37\% for PLA. Li et al. (2002) assessed the mechanical properties for orthogonal ply test specimens using CLT. They observed a maximum of $7.1 \%$ deviation between experimental and CLT predicted elastic modulus. Song et al. (2017) reported that specimens tested out of the plane direction displayed brittle behavior and displayed more ductile when tested in-plane direction. Croccolo et al. (2013) derived an analytical model to assess the strength and stiffness of the printed part based on perimeters deposited around the part edge. Rodriguez et al. (2001) studied the mechanical properties of FDM based ABS parts with different mesostructure. They reported that FDM produced parts had elastic modulus ranging from 11 to $37 \%$ and tensile strength is 22 to $57 \%$ lower than ABS filament. Huang and Singamneni (2015) assessed the effect of mesostructure resulting from variable processing conditions on material properties using the experimental and analytical methods. Tensile strength and elastic modulus were decreased with increment in raster angle. Kulkarni and Dutta (1999) studied the part stiffness resulting due to different deposition strategies in the FDM process. They reported that a part printed with $0^{\circ} / 90^{\circ}$ raster path yields the optimum part stiffness. Zou et al. (2016) studied the elasticity and yielding behavior of FDM processed ABS. They established a constitutive model to determine the relationship between elastic constant and raster angles. Rodriguez et al. (2003) examined the tensile properties of FDM processed ABS for mesostructure configuration. They observed that elastic modulus was lower by 11 to $37 \%$ and strength lower by 22 to $57 \%$ compared to ABS filament. Somireddy and Czekanski (2017) used CLT to estimate the mechanical properties of the FDM processed laminate. They reported that results obtained using classic laminate theory and experiment results were in good agreement.

On the other hand, several researchers carried out an experimental study to understand the effect of the build parameters on the tensile properties of the FDM printed parts. Rankouhi et al. (2016) reported that parts having smaller layer thickness displayed better tensile strength. Ahn et al. (2002) suggested that layers should be printed along the loading direction with a negative air gap to enhance the tensile strength. Durugan and Ertan (2014) studied the influence of rater and build direction on the mechanical properties of FDM parts. Parts printed horizontally at $0^{\circ}$ raster orientation have better mechanical strength. Garg and Bhattacharya (2017) carried out an FEA analysis of FDM parts. FE analysis indicated that at $0^{\circ}$ raster angle, strain at fracture and elongation increases with increment in layer height and tensile strength is reduced with an increase in the layer height. Garg et al. (2019) examined the influence of raster angle on tensile and flexural strength of 3D printed specimens. Tensile and flexural strength was highest for parts printed at $0^{\circ}$ raster orientation compared to those printed at $90^{\circ}$. Sood et al. (2010) reported a smaller raster angle, negative air gap, and smaller layer height, resulting in higher mechanical strength of the FDM part. Akhoundi et al. (2019) assessed the influence of infill patterns on the tensile and flexural strength of FDM processed parts. Higher mechanical strength can be obtained with a concentric pattern and higher infill density. Somireddy et al. (2019) assessed the mechanical behavior of FFF printed bi-directional laminates. They found that the lamina layup sequence and layer height significantly affect the tensile properties of FFF laminates. Bellini and Guceri (2003) carried out the mechanical characterization of FDM fabricated parts. The mechanical strength of the FDM part was mostly dependent on building direction and infill strategy. Dave et al. (2021) studied the influence of infill strategy on the tensile strength of FDM parts. They observed that part in horizontal orientation with the concentric pattern exhibited better strength. Zhou et al. (2020) investigated the bonding effect of different polymer blend materials in the FDM process. They observed a linear relationship between porosity and bonding strength. Shanmugam et al. (2020) evaluated the fatigue properties of the 3D printed polymeric 
materials. They reported that defects and voids should be considered in the optimization of the FDM process as they are responsible for the stress concentration. Khosravani et al. (2020) suggested that defects, surface roughness, anisotropy and the similarity between actual components and specimens must be considered for the fracture characterization of 3D-printed materials.

It can be seen that FDM parts have anisotropy in the mechanical properties, even if the raw material is isotropic. The mechanical properties of the printed part are significantly influenced by layer height, raster width, part orientation, raster deposition, the gap between rasters, etc. Aforementioned literature shows that CLT is explored to predict the tensile strength of the FDM part as a function of the raster deposition, however capability of the CLT model to predict the tensile strength at the different mesostructure combination (i.e., a different combinations of layer height and raster width) is still unrevealed. In this study, the development of a constitutive model has been attempted to predict the tensile properties with respect to various deposition sequences at the different configurations of raster width and layer height. The effect of the various configurations of raster width and layer height on the elastic constant required to develop a constitutive model has also been investigated. Initially, to apply CLT, unidirectional laminate has been printed and tested to obtain modulus of elasticity of printed laminate at the different configurations of layer height and raster width. Further, the classic laminate theory has been employed to estimate the tensile properties of FDM laminate and it has been validated using experimental results of FDM specimens with bi-directional raster depositions.

Section 2 includes a detailed description of the classic laminate theory (CLT) reported in the literature (Jones, 2014; Kaw, 2005; Somireddy and Czekanski, 2017). A method of estimating an elastic constant to be incorporated in the CLT is described in section 3. Correspondingly, the effect of mesostructure on the elastic constant for CLT and results obtained through CLT are discussed in detail in section 4 . The summary of the present work is outlined in the last section.

\section{Classic Laminate Theory (CLT)}

CLT is a predictive tool used to analyze the coupling effect, which may occur in composite laminates. With the help of CLT, it is possible to predict strains, displacements, and curvatures generated within the laminate as a function of mechanical loading. Since FDM printed parts similar to laminate structures, they can be evaluated by the CLT (Jones, 2014; Kaw, 2005; Somireddy \& Czekanski, 2017). The constitutive relation for a lamina is as follows:

$$
\left\{\begin{array}{l}
\sigma_{11} \\
\sigma_{22} \\
\tau_{12}
\end{array}\right\}=\left[\begin{array}{ccc}
Q_{11} & Q_{12} & 0 \\
Q_{12} & Q_{22} & 0 \\
0 & 0 & Q_{66}
\end{array}\right]\left\{\begin{array}{l}
\varepsilon_{11} \\
\varepsilon_{22} \\
\gamma_{12}
\end{array}\right\}
$$

The terms in the matrix are

$$
\begin{aligned}
& Q_{11}=\frac{E_{1}}{1-v_{12} v_{21}}, Q_{12}=\frac{v_{21} E_{1}}{1-v_{12} v_{21}} Q_{22}=\frac{E_{2}}{1-v_{12} v_{21}}, \\
& Q_{66}=G_{12} \text { with } \\
& v_{21}=v_{12} \frac{E_{2}}{E_{1}}
\end{aligned}
$$

The resultant force and moment per unit width in the laminate with $\mathrm{N}$ numbers of layers are written as

$$
\begin{aligned}
& \left\{\begin{array}{l}
N_{x x} \\
N_{y y} \\
N_{x y}
\end{array}\right\}=\int_{-h / 2}^{h / 2}\left\{\begin{array}{l}
\sigma_{x x} \\
\sigma_{y y} \\
\sigma_{x y}
\end{array}\right\} d z=\sum_{K=1}^{N} \int_{h_{k}}^{h_{k+1}}\left\{\begin{array}{l}
\sigma_{x x} \\
\sigma_{y y} \\
\tau_{x y}
\end{array}\right\} d z,\{N\}=\sum_{K=1}^{N} \int_{h_{k}}^{h_{k+1}}\{\sigma\} d z \\
& \left\{\begin{array}{l}
M_{x x} \\
M_{y y} \\
M_{x y}
\end{array}\right\}=\int_{-h / 2}^{h / 2}\left\{\begin{array}{l}
\sigma_{x x} \\
\sigma_{y y} \\
\sigma_{x y}
\end{array}\right\} d z=\sum_{K=1}^{N} \int_{h_{k}}^{h_{k+1}}\left\{\begin{array}{l}
\sigma_{x x} \\
\sigma_{y y} \\
\tau_{x y}
\end{array}\right\} d z,\{M\}=\sum_{K=1}^{N} \int_{h_{k}}^{h_{k+1}}\{\sigma\} z d z
\end{aligned}
$$

Using Eqs. (1-3) we have,

$$
\begin{aligned}
& \{N\}=\sum_{K=1}^{N}[\bar{Q}]\left[\int_{h_{k}}^{h_{k+1}}\left\{\mathcal{E}^{0}\right\} d z+\int_{h_{k}}^{h_{k+1}}\{k\} z d z\right] \\
& \{N\}=[A]\left\{\mathcal{E}^{0}\right\}+[B]\{k\}
\end{aligned}
$$




$$
\begin{aligned}
& \{M\}=\sum_{K=1}^{N}[\bar{Q}]\left[\int_{h_{k}}^{h_{k+1}}\left\{\varepsilon^{0}\right\} z d z+\int_{h_{k}}^{h_{k+1}}\{k\} z^{2} d z\right] \\
& \{M\}=[B]\left\{\varepsilon^{0}\right\}+[D]\{k\}
\end{aligned}
$$

where $N_{x x}$ is the normal force per unit width in the x-direction,

$N_{y y}$ is the normal force per unit width in the y-direction,

$N_{x y}$ is a shear force,

$M_{x x}$ is the bending moment yz plane,

$M_{y y}$ is the bending moments in the xz planes, and

$M_{x y}$ is the twisting moment.

Matrix $[A]$ denotes the in-plane stiffness, matrix $[B]$ denotes the bending stiffness coupling and $[D]$ denotes the bending stiffness. The matrices $[\mathrm{A}]$, [B], and [D] can be written as follows:

$$
\begin{aligned}
& {[A]=\sum_{k=1}^{N}[\bar{Q}] \int_{h_{k}}^{h_{k+1}} d z} \\
& {[B]=\sum_{k=1}^{N}[\bar{Q}] \int_{h_{k}}^{h_{k+1}} z d z} \\
& {[D]=\sum_{k=1}^{N}[\bar{Q}] \int_{h_{k}}^{h_{k+1}} z^{2} d z}
\end{aligned}
$$

In the case of symmetric laminate, there is no extension bending coupling because of the coupling matrix $[\mathrm{B}]=0$. Then, the strains for a symmetric laminate are given from equation (4) as

$$
\left\{\begin{array}{l}
\varepsilon_{x x}^{0} \\
\varepsilon_{y y}^{0} \\
\gamma_{x y}^{0}
\end{array}\right\}=[A]^{-1}\left\{\begin{array}{l}
N_{x x} \\
N_{y y} \\
N_{x y}
\end{array}\right\}
$$

In the uniaxial tensile test, the tensile loading is parallel to $\mathrm{x}$-direction of laminate for laminate thickness $\mathrm{h}$, then the inplane forces for this load case become $N_{x x}=h \sigma_{x x}, N_{y y}=0$ and $N_{x y}=0$. The stress-strain relation for the uniaxial tensile test is $\sigma_{x x}=E_{x x} \varepsilon_{x x}^{0}$, using these relationships the modulus of elasticity in the x-direction is determined as follows

$$
E_{x x}=\frac{1}{\left[A^{-1}\right] h}
$$

To determine $E_{x x}$ of the laminate, it is necessary to have the lamina's elastic modulus such as $E_{1}, E_{2}, G_{12}$ and $v_{12}$.

The laminate consecutive equation is expressed as follows:

$\left\{\begin{array}{l}N \\ M\end{array}\right\}=\left[\begin{array}{ll}A & B \\ B & D\end{array}\right]\left\{\begin{array}{c}\varepsilon^{0} \\ \kappa\end{array}\right\}$

For a test specimen in tension only non zero elements of $\mathrm{N}$ and $\mathrm{M}$ is $N_{x x}$. Thus, equation (13) is simplified to

$$
\left\{\begin{array}{c}
N \\
0
\end{array}\right\}=\left[\begin{array}{ll}
A & B \\
B & D
\end{array}\right]\left\{\begin{array}{c}
\varepsilon^{0} \\
\kappa
\end{array}\right\}
$$

where,

$$
N=\left[\begin{array}{c}
N_{x x} \\
0 \\
0
\end{array}\right]
$$

The strain is computed as

$$
\varepsilon^{0}=A_{11}^{*} N_{x x}
$$




\section{Elastic Constant Estimation}

An FDM based 3D printer (as shown in Fig. 1) equipped with marlin firmware and a nozzle with a diameter of $0.4 \mathrm{~mm}$ was used for the fabrication of the test specimen. Four printing parameters (scan speed, No. of perimeters, \% infill and infill patterns) were held constant during the fabrication of test samples and are listed in Table 1 . The bed temperature was set at $70{ }^{\circ} \mathrm{C}$ and the nozzle temperature at $210{ }^{\circ} \mathrm{C}$ for PLA material. As discussed earlier, four elastic constants are required to analyze an orthotropic lamina under plane stress. These properties can be obtained through the tensile test on the specimen with a raster angle of $0^{\circ}, 90^{\circ}$ and $45^{\circ}$ at the different configurations of layer height and raster width.

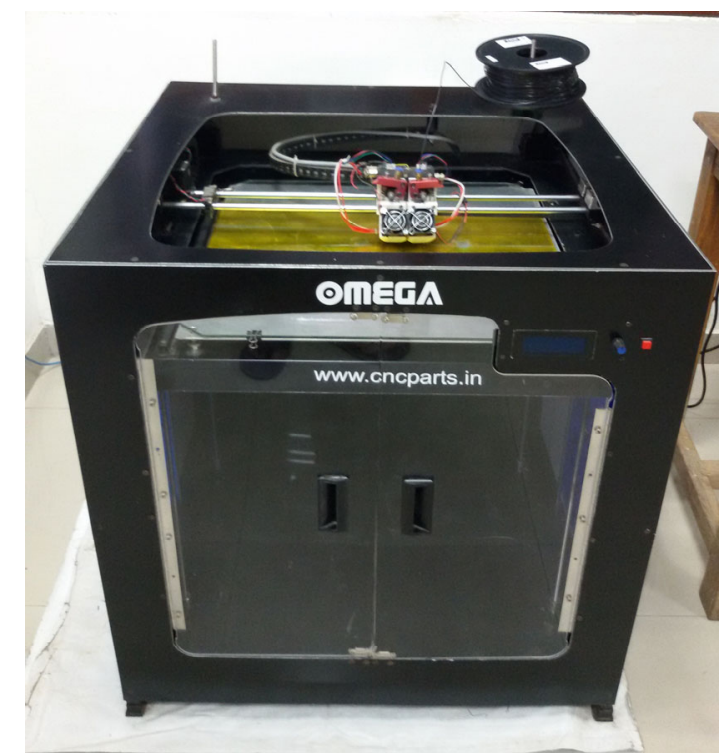

Fig. 1. FDM based 3D printer

Longitudinal modulus of elasticity $E_{1}$ and poison ratio $V_{12}$ are determined using a longitudinal specimen $\left(0^{\circ}\right)$ and transverse elastic modulus $E_{2}$ is determined using a transverse specimen $\left(90^{\circ}\right)$. Specimen with raster orientation $\theta=45^{\circ}$ is used to determine elastic modulus $E_{45}$ that is used to calculate the shear modulus of $G_{12}$ :

$$
G_{12}=\left[\frac{4}{E_{45}}-\frac{1}{E_{1}}-\frac{1}{E_{2}}+\frac{2 v_{12}}{E_{1}}\right]^{-1}
$$

Eq. (17) was used along with other experimentally obtained elastic constants $E_{1} E_{2}$ and $V_{12}$ to obtain the in-plane shear modulus $G_{12}$.

$\underline{\text { Table 1. Constant and variable parameter during } 3 \mathrm{D} \text { printing }}$

\begin{tabular}{|c|c|c|c|c|c|c|}
\hline \multirow[b]{2}{*}{ Fixed parameter } & \multirow[b]{2}{*}{ Value } & \multirow[b]{2}{*}{ Variable parameter } & \multicolumn{4}{|c|}{ Value } \\
\hline & & & Level 1 & Level 2 & Level 3 & $\begin{array}{c}\text { Level } \\
4 \\
\end{array}$ \\
\hline Scan speed & $50 \mathrm{~mm} / \mathrm{s}$ & Layer height $\left(\mathrm{h}_{\mathrm{L}}\right)(\mathrm{mm})$ & 0.1 & 0.2 & 0.3 & - \\
\hline No. of perimeters & 1 & Raster width (WR) (mm) & 0.4 & 0.5 & 0.6 & 0.7 \\
\hline$\%$ infill & $100 \%$ & Raster angle $\left(\theta_{R}\right)\left({ }^{\circ}\right)$ & 0 & 45 & 90 & - \\
\hline
\end{tabular}
Infill pattern rectilinear

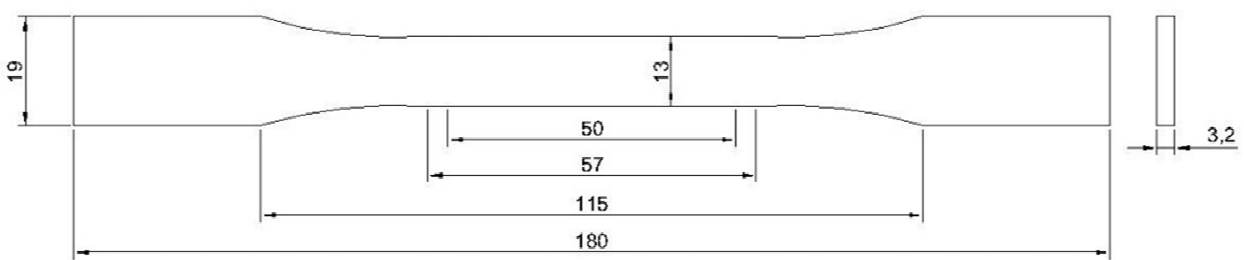

Fig. 2. Tensile specimen as per ASTM D638 (all dimensions are in mm)

As shown in Table 1, twelve different configurations of layer height and raster width were considered and tested to investigate the effect of the filament cross-section on tensile properties. For all the possible combinations of layer height and 
raster width, test samples were printed at the three different raster angles of $0^{\circ}, 45^{\circ}$ and $90^{\circ}$ to obtain modulus of elasticity in the longitudinal direction and transverse direction and shear modulus of the 3D printed specimens. In the present investigation, ASTM D638 standard was selected to print the standard tensile test sample as depicted in Fig 2.

For tensile test fabrication, the first 3D model was created for test samples following ASTM D638 standard, using CAD software. It is then exported as a STL file and subsequently loaded into slicing software. In slicing software, all the printing parameters were adjusted and the set of printing instructions was exported as a GCode file. Then the GCode file was loaded in an FDM based 3D printing machine to print the unidirectional FDM component. Unidirectional test specimens with $0^{\circ}$, $90^{\circ}$, and $45^{\circ}$ raster orientations were fabricated to determine elastic constants at the different configurations of layer height and raster width as discussed earlier. In order to compare the experimental and CLT results, specimens with $0^{\circ} / 90^{\circ}, 30^{\circ} /-60^{\circ}$, and $45^{\circ} /-45^{\circ}$ raster orientation were also fabricated and tested for different configurations of layer height and raster width.

PLA specimens were tested at room temperature under displacement control on a Tinius Olsen H50KL universal test machine, with computer control and data acquisition. The displacement rate was set to $5 \mathrm{~mm} / \mathrm{min}$ according to standard and tested until failure occurred.

\section{Results and Discussions}

The result data were processed to determine the modulus of elasticity and the ultimate tensile strength. The tensile properties for different configurations of layer height and raster width were determined experimentally and are plotted in Fig 3.

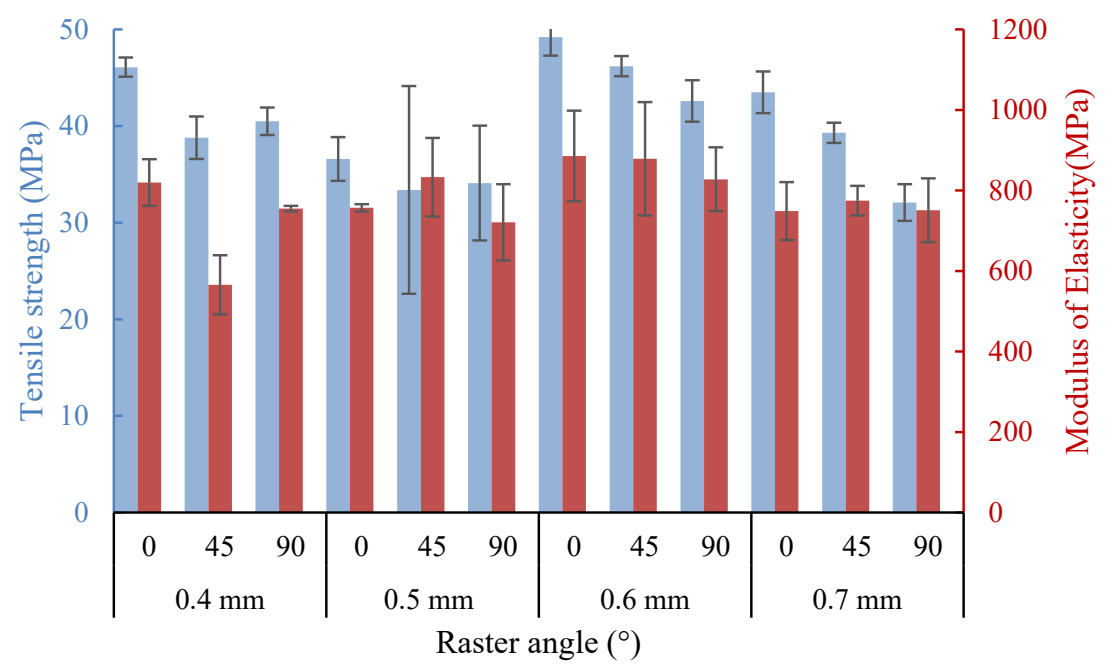

(a)

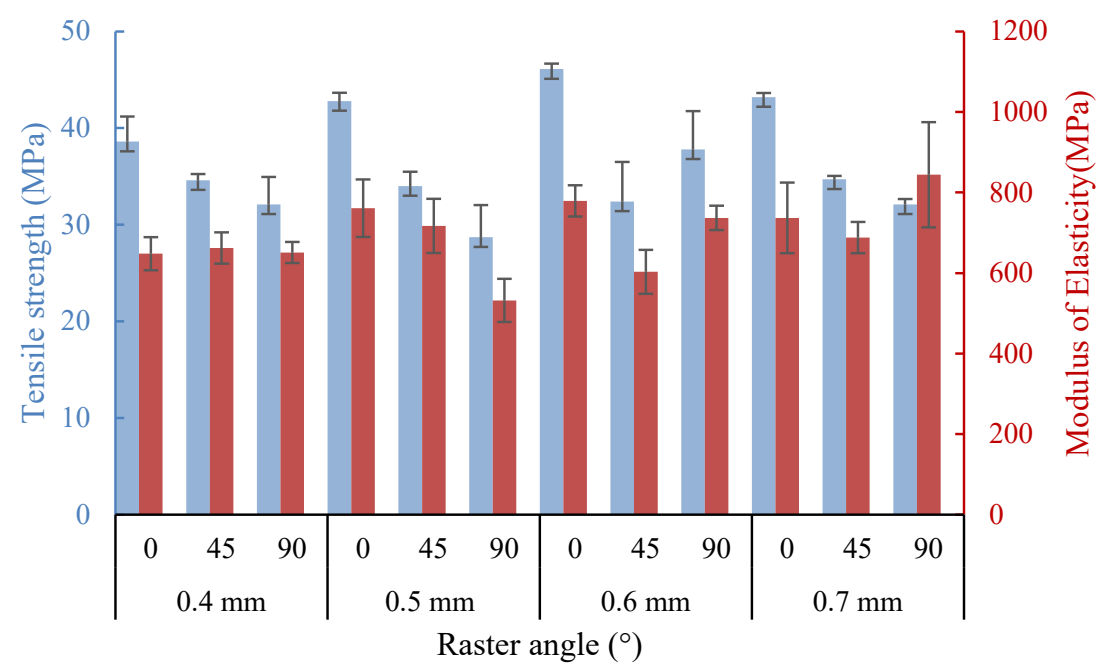

(b) 


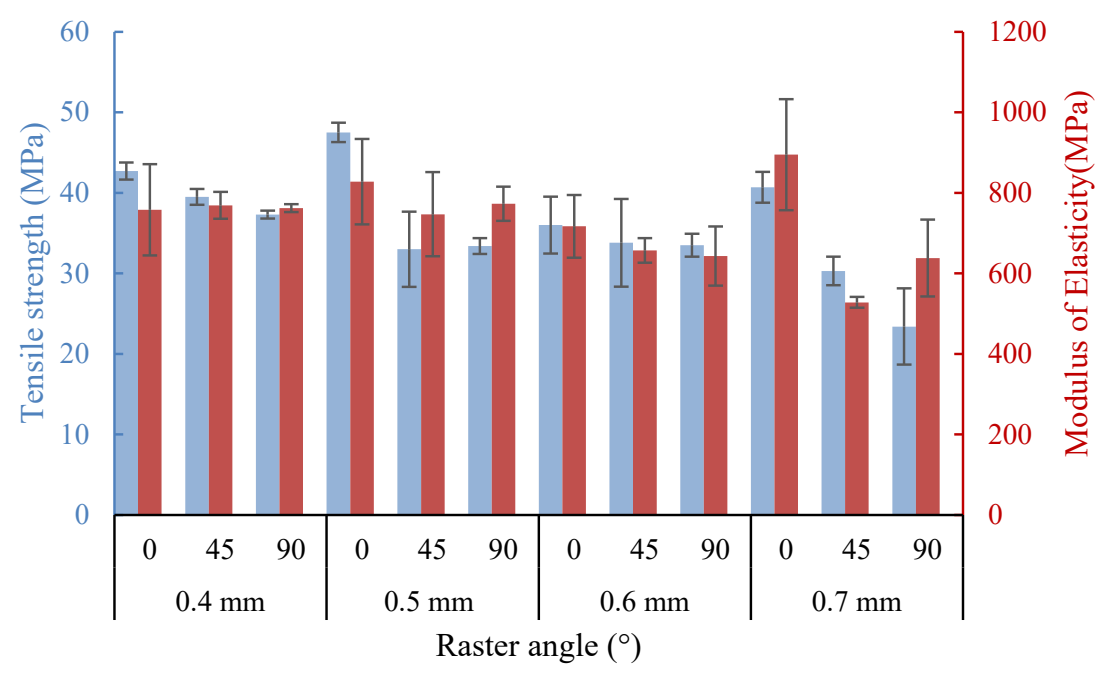

(c)

Fig. 3. Tensile properties at different raster angle for different configuration of layer height and raster width (a) $0.1 \mathrm{~mm}$ layer height, (b) $0.2 \mathrm{~mm}$ layer height and (c) $0.3 \mathrm{~mm}$ layer height

Fig. 3 shows that parts printed with a combination of layer height of $0.1 \mathrm{~mm}$ and raster width of $0.4 \mathrm{~mm}$ displayed higher tensile properties and with a combination of layer height of $0.3 \mathrm{~mm}$ and raster width of 0.7 displayed lower tensile properties. Lower layer height and raster width give a higher bonding area between adjacent raster and layers, resulting in higher bonding strength between layers with a lesser amount of voids. Higher stiffness can also be obtained due to a larger bonding area capable of withstanding a higher loading. It may be due to the fact that at lower layer height, higher extrusion pressure may help to form a quick and strong bond that results in higher strength.

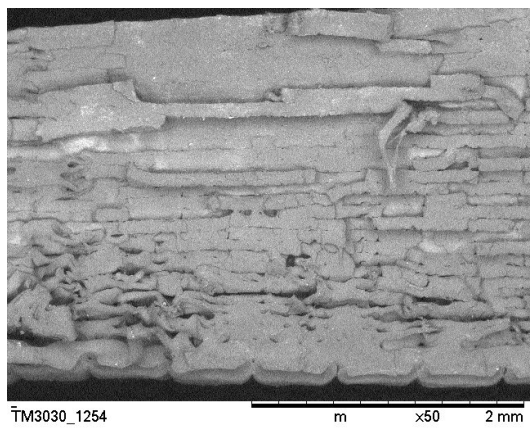

(a)

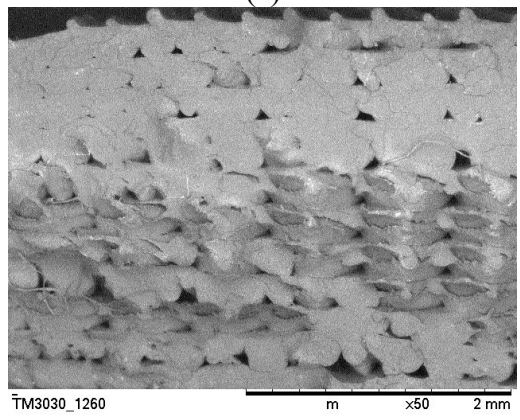

(c)

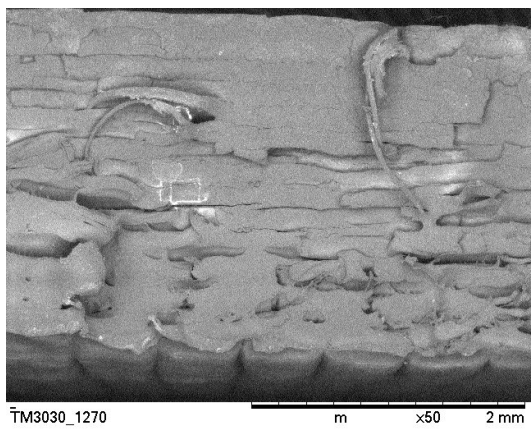

(b)

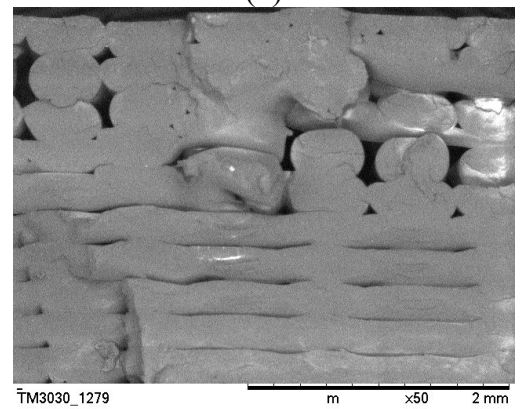

(d)

Fig. 4. SEM images for fractured surface of test specimen at (a) $0.1 \mathrm{~mm}$ layer height- $0.4 \mathrm{~mm}$ raster width, (b) 0.1 $\mathrm{mm}$ layer height $-0.7 \mathrm{~mm}$ raster width, (c) $0.3 \mathrm{~mm}$ layer height $-0.4 \mathrm{~mm}$ raster width, (d) $0.3 \mathrm{~mm}$ layer height -0.7 $\mathrm{mm}$ raster width

Further, lower raster width time taken for printing is higher, allowing more heat dissipation that improves the diffusion between adjacent layers and rasters and may result in a stronger bond formation. These resulted in higher tensile strength and higher stiffness. At higher layer height and raster width, bonding area between layers decreases due to voids that result in reduced bonding strength. The reduced bonding strength leads to a reduction in strength and stiffness of the printed part. It 
can also be noticed that at higher raster width, the effective bonding area was found to be decreased due to the number of voids that reduce the strength and stiffness of the fabricated part.

Fig. 4 shows the fractured surface of the test specimen at the different configurations of layer height and raster width. It can be seen that a higher bonding area is observed at $0.1 \mathrm{~mm}$ layer height and $0.4 \mathrm{~mm}$ raster width that help to achieve higher strength. On the other hand, at a combination of $0.3 \mathrm{~mm}$ layer height and $0.7 \mathrm{~mm}$ raster width, a large number of voids and a significant reduction in bonding area were observed, which might be a reason for a decrease in strength. The results confirm that mesostructure is one of the critical aspects that can affect the tensile strength and modulus of elasticity of the fabricated part.

From Fig 3, it is also depicted that higher tensile strength is achieved at $0^{\circ}$ raster angle and it is decreased with increment in raster angle. At $0^{\circ}$ raster angle, all the layers are deposited parallel to tensile loading capable of withstanding higher loading resulting in higher strength and stiffness. While at $90^{\circ}$ raster angle, all the layers are deposited perpendicular to the tensile loading. Tensile strength is dependent on the bonding between adjacent rasters in perpendicular layer arrangement, bonding between rasters is always weaker than continuous fiber that is resulting in a lesser tensile strength. The stress-strain curve has been obtained at varying raster angles as shown in Fig. 5 at different configurations of layer height and raster width. It can be seen that specimens printed with $0^{\circ}$ raster angle displayed ductile failure due to a significant amount of pulling and necking of individual rasters during failure. On the other hand, at $45^{\circ}$ and $90^{\circ}$ raster angles, specimens displayed brittle failure. The brittle failure took place through the bonding between the adjacent rasters that is always weaker than the monofilament, which leads to abrupt failure with less or no elongation. In general, it can be stated that at $0^{\circ}$ raster angle the printed part has a ductile failure with little plastic behavior compared to the $90^{\circ}$ raster angle printed part, which displayed brittle failure with no plastic behavior. The results confirm that raster angle is one of the most important process parameters that govern the tensile strength, modulus of elasticity and mode of fracture. Similar observations have also been reported by casavola et al. (2016) and Somireddy and Czekanski (2017).
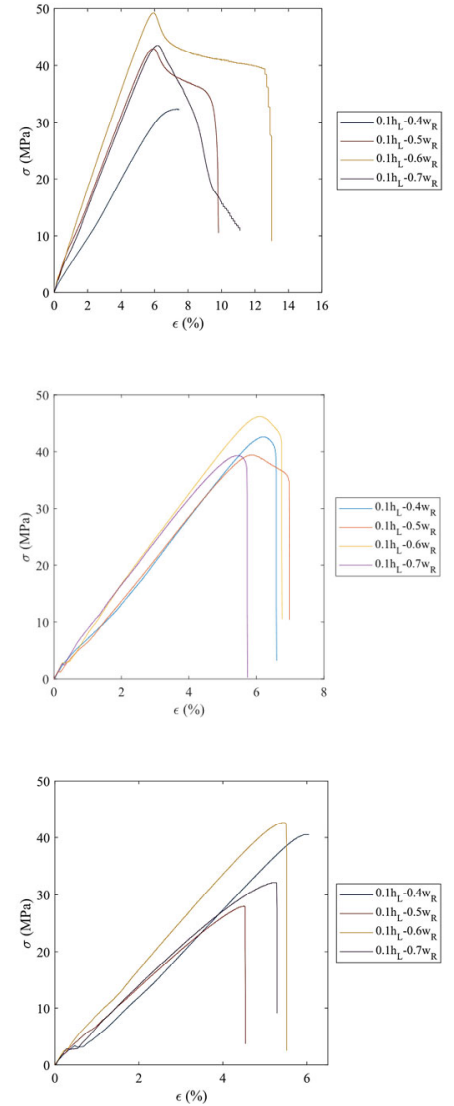

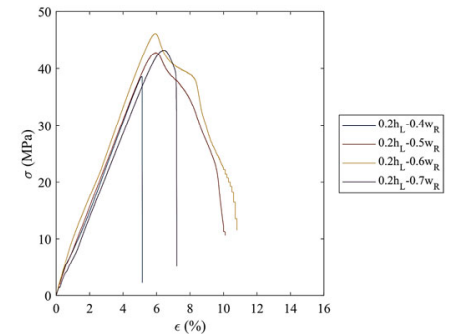

(a)

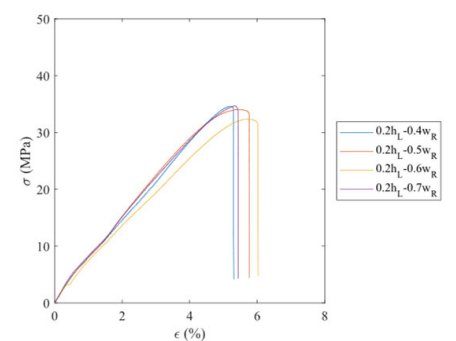

(b)

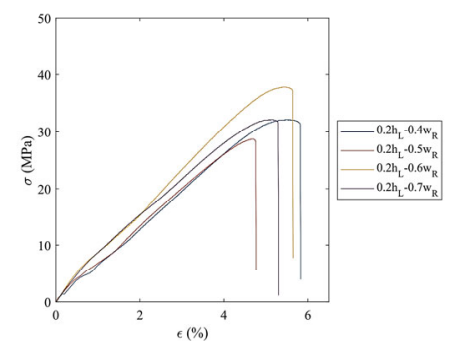

(c)
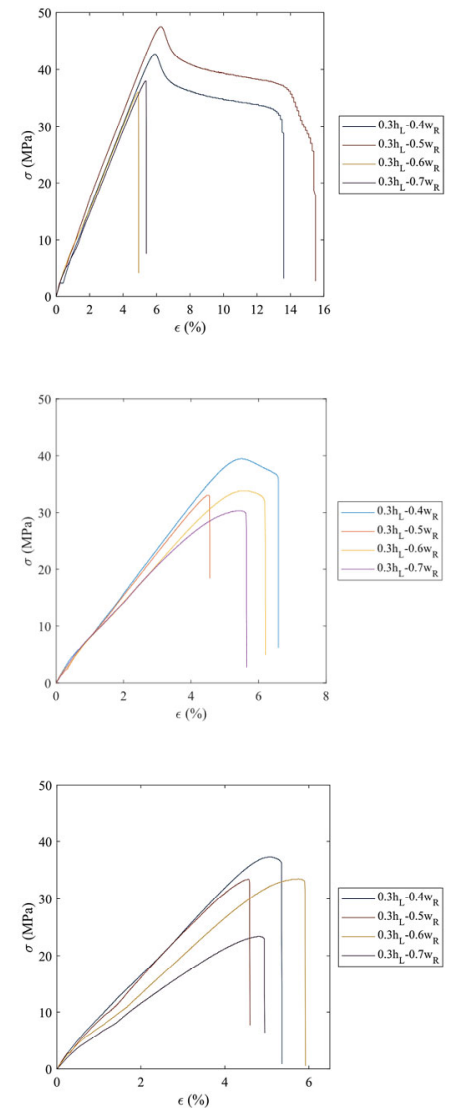

Fig. 5. Stress vs. strain diagram at (a) $0^{\circ}$, (b) $45^{\circ}$ and (c) $90^{\circ}$ raster angle 
Table 2. Elastic constant to be used in CLT at the different configuration of layer height and raster width.

\begin{tabular}{|c|c|c|c|c|}
\hline Layer height & \multicolumn{4}{|c|}{$0.1 \mathrm{~mm}$} \\
\hline Raster width & $0.4 \mathrm{~mm}$ & $0.5 \mathrm{~mm}$ & $0.6 \mathrm{~mm}$ & $0.7 \mathrm{~mm}$ \\
\hline$E_{1}$ & $820 \mathrm{MPa}$ & $757 \mathrm{MPa}$ & $886 \mathrm{MPa}$ & $749 \mathrm{MPa}$ \\
\hline$E_{2}$ & $755 \mathrm{MPa}$ & $721 \mathrm{MPa}$ & $828 \mathrm{MPa}$ & $751 \mathrm{MPa}$ \\
\hline$G_{12}$ & $187 \mathrm{MPa}$ & $334 \mathrm{MPa}$ & $335 \mathrm{MPa}$ & $294 \mathrm{MPa}$ \\
\hline$v_{12}$ & 0.34 & 0.34 & 0.34 & 0.34 \\
\hline Layer height & \multicolumn{4}{|c|}{$0.2 \mathrm{~mm}$} \\
\hline Raster width & $0.4 \mathrm{~mm}$ & $0.5 \mathrm{~mm}$ & $0.6 \mathrm{~mm}$ & $0.7 \mathrm{~mm}$ \\
\hline$E_{1}$ & $648 \mathrm{MPa}$ & $761 \mathrm{MPa}$ & $779 \mathrm{MPa}$ & $737 \mathrm{MPa}$ \\
\hline$E_{2}$ & $651 \mathrm{MPa}$ & $532 \mathrm{MPa}$ & $737 \mathrm{MPa}$ & $844 \mathrm{MPa}$ \\
\hline$G_{12}$ & $249 \mathrm{MPa}$ & $305 \mathrm{MPa}$ & $205 \mathrm{MPa}$ & $238 \mathrm{MPa}$ \\
\hline$v_{12}$ & 0.34 & 0.34 & 0.34 & 0.34 \\
\hline Layer height & \multicolumn{4}{|c|}{$0.3 \mathrm{~mm}$} \\
\hline Raster width & $0.4 \mathrm{~mm}$ & $0.5 \mathrm{~mm}$ & $0.6 \mathrm{~mm}$ & $0.7 \mathrm{~mm}$ \\
\hline$E_{1}$ & $758 \mathrm{MPa}$ & $828 \mathrm{MPa}$ & $717 \mathrm{MPa}$ & $895 \mathrm{MPa}$ \\
\hline$E_{2}$ & $762 \mathrm{MPa}$ & $773 \mathrm{MPa}$ & $643 \mathrm{MPa}$ & $638 \mathrm{MPa}$ \\
\hline$G_{12}$ & $288 \mathrm{MPa}$ & $272 \mathrm{MPa}$ & $244 \mathrm{MPa}$ & $177 \mathrm{MPa}$ \\
\hline$v_{12}$ & 0.34 & 0.34 & 0.34 & 0.34 \\
\hline
\end{tabular}

Table 2 shows the elastic constant required to characterize the tensile properties of FDM printed parts. These constants have been used as an input in the CLT model based on which tensile properties of specimens with $0^{\circ} / 90^{\circ}, 30^{\circ} /-60^{\circ}$, and $45^{\circ} /-$ $45^{\circ}$ raster angle have been predicted. Test specimens at $0^{\circ} / 90^{\circ}, 30^{\circ} /-60^{\circ}$, and $45^{\circ} /-45^{\circ}$ raster angle were printed at the different configurations of layer height and raster width. Then after the tensile test was carried out to obtain tensile strength of the printed specimens. Further, experimental results were compared with the results obtained through CLT calculation to validate the CLT calculation. The calculated and experimental tensile strength results are plotted against the raster angle as shown in Fig. 6 for all different configurations of layer height and raster width. In general, it is seen that experimental results are in good agreement with CLT calculations. The tensile strength increases with an increment in raster angle for different test conditions. It can be seen that the highest tensile strength is obtained at $45^{\circ} /-45^{\circ}$ raster angle followed by $30^{\circ} /-60^{\circ}$ and $0^{\circ} / 90^{\circ}$ raster angle. Higher tensile strength has been obtained at a combination of $0.1 \mathrm{~mm}$ layer height and $0.7 \mathrm{~mm}$ raster width. In contrast, lower tensile strength has been obtained at a combination of $0.2 \mathrm{~mm}$ layer height and $0.4 \mathrm{~mm}$ raster width. It may be due to a higher bonding area at $0.1 \mathrm{~mm}$ layer height and $0.4 \mathrm{~mm}$ raster width that help to achieve higher strength. On the other hand, at a combination of $0.3 \mathrm{~mm}$ layer height and $0.7 \mathrm{~mm}$ raster width, a large number of voids and a significant reduction in bonding area may be observed, which might be a reason for a decrease in strength. Casavola et al. (2016) observed good agreement in CLT and experimental results. Somireddy and Czekanski (2017) also predicted the tensile properties with different stacking sequences using CLT and found them to be in good agreement with experimental results. It is seen from Fig 6 that the classic laminate theory results are comparable with that of experimental results and therefore the classic laminate theory could be used to characterize the tensile behavior of the FDM parts. 

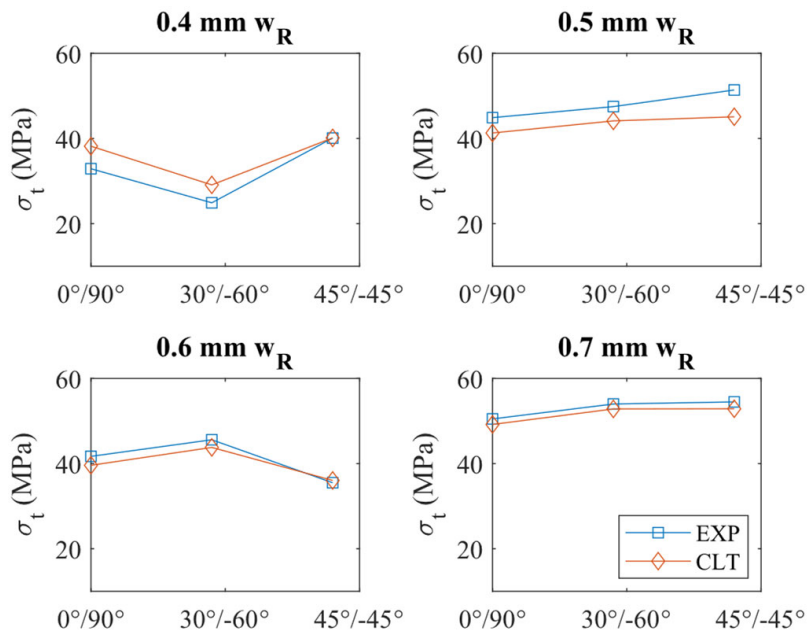

(a)
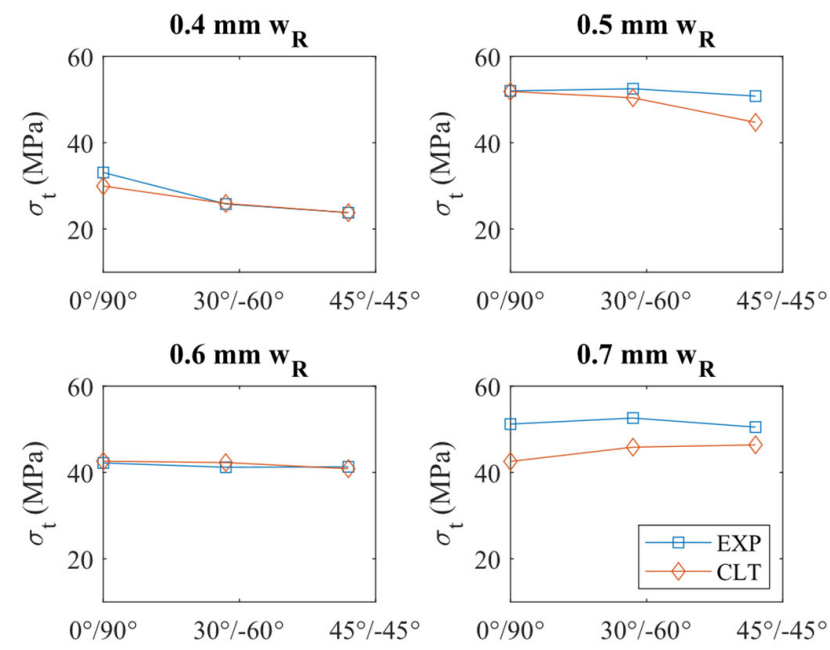

(b)
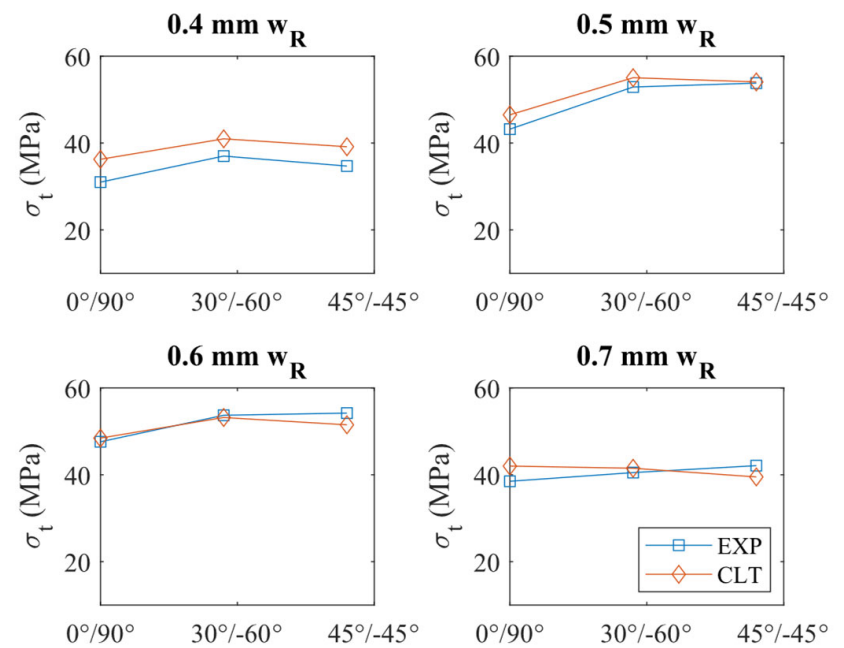

(c)

Fig. 6. Comparison between experimental and CLT calculated tensile strength for (a) $0.1 \mathrm{~mm}$ layer height, (b) $0.2 \mathrm{~mm}$ layer height and (c) $0.3 \mathrm{~mm}$ layer height 
Fig. 7 shows the fractured surface of the test sample at various raster angles. At $45^{\circ} / 45^{\circ}$ raster angle, layers have been deposited at $45^{\circ}$ to the loading direction then the next layer deposited with a $90^{\circ}$ increment to the previously deposited layer. The bonding between the layer and within the layer is very effective, which increases the tensile strength of the part. The tensile performance depends upon the quality of the bonding formation between the rasters as well as between the layers. Tensile performance could be improved with higher quality of bonding between rasters and layers It can be seen that, for $0^{\circ} / 90^{\circ}$ raster angle, half of the layers are deposited longitudinal to loading direction and half of the layers are deposited transversely to the loading direction. Therefore, half of the layers have been pulled through the longitudinal direction, but the remaining layers have been pulled through the bonding between adjacent beads, which lowers the strength of the parts. The bonding area between the layers and within the layers is comparatively decreased due to presence of porosity, reducing the strength of the printed part. The presence of the porosity has an adverse effect on the tensile performance of the printed part as it may lead to catastrophic failure. Zhou et al. (2020) have also observed the reduction in the mechanical performance of printed parts with the appearance of the porosity in the part.

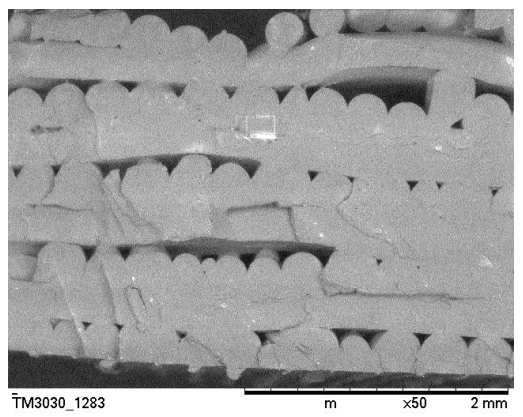

(a)

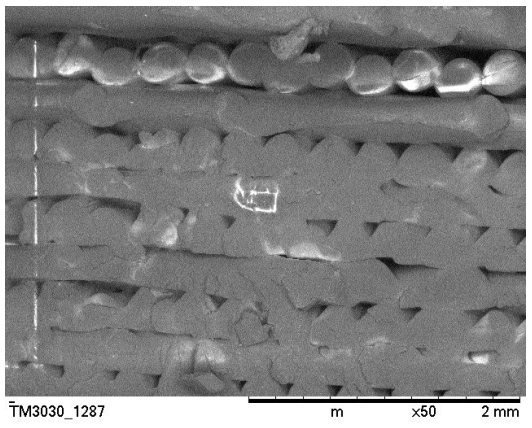

(b)

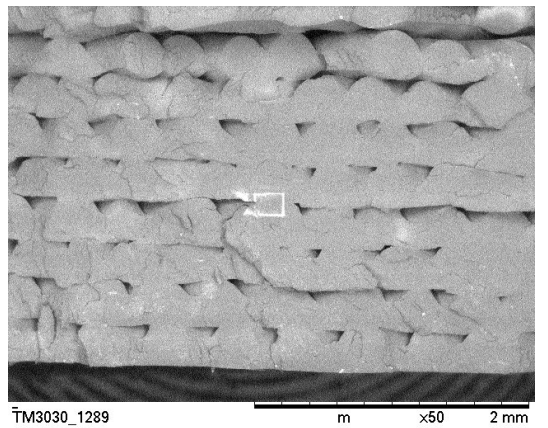

(c)

Fig. 7. SEM image for the fractured surface of the test specimen at (a) $0^{\circ} / 90^{\circ}$, (b) $30^{\circ} /-60^{\circ}$ and (c) $45^{\circ} /-45^{\circ}$

\section{Conclusions}

In the present investigation, the effect of mesostructure configuration and raster angle on the tensile strength of FDM printed PLA parts has been evaluated. Twelve different mesostructure configurations have been tested to investigate their influence on the tensile properties of the printed part. Experiments were carried out at various mesostructure configurations to find out the tensile strength and elastic modulus. Effect of the layer height, raster width and raster angle on the tensile strength and elastic modulus of the printed parts have been investigated. Lower layer height and raster width help to achieve higher tensile strength and elastic modulus. Moreover, higher tensile strength and stiffness are achieved at the $0^{\circ}$ raster angle due to parallel alignment of fibers while at $90^{\circ}$ raster angle lower strength and stiffness are obtained. Elastic modulus obtained through unidirectional FDM printed laminate was used as the input in the classic laminate theory. Further, Classic laminate theory was applied to predict the tensile behavior of the FDM printed bi-directional specimens and the results obtained through CLT calculation are validated using experimental results. Experimental tensile results are in good agreement with those obtained by CLT calculations. Hence, the classic laminate theory (CLT) is able to predict the tensile properties of FDM printed parts accurately.

\section{References}

Ahn, S. H., Montero, M., Odell, D., Roundy, S., \& Wright, P. K. (2002). Anisotropic material properties of fused deposition modeling ABS. Rapid Prototyping Journal, 8, 248-257.

Akhoundi, B., \& Behravesh, A. H. (2019). Effect of filling pattern on the tensile and flexural mechanical properties of FDM 3D printed products. Experimental Mechanics, 59(6), 883-897.

Alaimo, G., Marconi, S., Costato, L., \& Auricchio, F. (2017). Influence of meso-structure and chemical composition on FDM 3D-printed parts. Composites Part B: Engineering, 113, 371-380.

Ameri, B., Taheri-Behrooz, F., \& Aliha, M. R. M. (2020). Fracture loads prediction of the modified 3D-printed ABS specimens under mixed-mode I/II loading. Engineering Fracture Mechanics, 235, 107181.

Ameri, B., Taheri-Behrooz, F., \& Aliha, M. R. M. (2021). Evaluation of the Geometrical Discontinuity effect on MixedMode I/II Fracture Load of FDM 3D-Printed Parts. Theoretical and Applied Fracture Mechanics, 113, 102953.

Bellini, A., \& Güçeri, S. (2003). Mechanical characterization of parts fabricated using fused deposition modeling. Rapid Prototyping Journal, 9, 252-264

Casavola, C., Cazzato, A., Moramarco, V., \& Pappalettere, C. (2016). Orthotropic mechanical properties of fused deposition modelling parts described by classical laminate theory. Materials \& Design, 90, 453-458.

Croccolo, D., De Agostinis, M., \& Olmi, G. (2013). Experimental characterization and analytical modelling of the mechanical behaviour of fused deposition processed parts made of ABS-M30. Computational Materials Science, 79, $506-518$. 
Dave, H. K., Patadiya, N. H., Prajapati, A. R., \& Rajpurohit, S. R. (2021). Effect of infill pattern and infill density at varying part orientation on tensile properties of fused deposition modeling-printed poly-lactic acid part. Proceedings of the Institution of Mechanical Engineers, Part C: Journal of Mechanical Engineering Science, 235(10), 1811-1827.

Durgun, I., \& Ertan, R. (2014). Experimental investigation of FDM process for improvement of mechanical properties and production cost. Rapid Prototyping Journal, 20, 228-235.

Garg, A., \& Bhattacharya, A. (2017). An insight to the failure of FDM parts under tensile loading: finite element analysis and experimental study. International Journal of Mechanical Sciences, 120, 225-236.

Garg, A., Bhattacharya, A., \& Batish, A. (2017). Failure investigation of fused deposition modelling parts fabricated at different raster angles under tensile and flexural loading. Proceedings of the Institution of Mechanical Engineers, Part B: Journal of Engineering Manufacture, 231(11), 2031-2039.

Huang, B., \& Singamneni, S. (2015). Raster angle mechanics in fused deposition modelling. Journal of Composite Materials, 49(3), 363-383.

Jones, R.M., (2014). Mechanics of composite materials. CRC Press.

Kaw, A.K., (2005). Mechanics of composite materials. CRC Press.

Khosravani, M. R., Berto, F., Ayatollahi, M. R., \& Reinicke, T. (2020). Fracture behavior of additively manufactured components: A review. Theoretical and Applied Fracture Mechanics, 109, 102763.

Kulkarni, P., \& Dutta, D. (1999). Deposition strategies and resulting part stiffnesses in fused deposition modeling, Journal of Manufacturing Science and Engineering, 121, 93-103

Li, L., Sun, Q., Bellehumeur, C., \& Gu, P. (2002). Composite modeling and analysis for fabrication of FDM prototypes with locally controlled properties. Journal of manufacturing processes, 4(2), 129-141.

Rajpurohit, S. R., \& Dave, H. K. (2018). Flexural strength of fused filament fabricated (FFF) PLA parts on an open-source 3D printer. Advances in Manufacturing, 6(4), 430-441.

Rajpurohit, S. R., \& Dave, H. K. (2019). Analysis of tensile strength of a fused filament fabricated PLA part using an opensource 3D printer. The International Journal of Advanced Manufacturing Technology, 101(5), 1525-1536.

Rankouhi, B., Javadpour, S., Delfanian, F., \& Letcher, T. (2016). Failure analysis and mechanical characterization of 3D printed ABS with respect to layer thickness and orientation. Journal of Failure Analysis and Prevention, 16(3), 467-481.

Rodríguez, J. F., Thomas, J. P., \& Renaud, J. E. (2001). Mechanical behavior of acrylonitrile butadiene styrene (ABS) fused deposition materials. Experimental investigation. Rapid Prototyping Journal, 7, 148-158.

Rodríguez, J. F., Thomas, J. P., \& Renaud, J. E. (2003). Mechanical behavior of acrylonitrile butadiene styrene fused deposition materials modeling. Rapid Prototyping Journal, 9, 219-230.

Shanmugam, V., Das, O., Babu, K., Marimuthu, U., Veerasimman, A., Johnson, D. J., Neisiany, R.E., Hedenqvist, M.S., Ramakrishna, S, \& Berto, F. (2021). Fatigue behaviour of FDM-3D printed polymers, polymeric composites and architected cellular materials. International Journal of Fatigue, 143, 106007.

Somireddy, M., \& Czekanski, A. (2017). Mechanical characterization of additively manufactured parts by FE modeling of mesostructure. Journal of Manufacturing and Materials Processing, 1(2), 18.

Somireddy, M., Singh, C. V., \& Czekanski, A. (2019). Analysis of the material behavior of 3D printed laminates via FFF. Experimental Mechanics, 59(6), 871-881.

Song, Y., Li, Y., Song, W., Yee, K., Lee, K. Y., \& Tagarielli, V. L. (2017). Measurements of the mechanical response of unidirectional 3D-printed PLA. Materials \& Design, 123, 154-164.

Sood, A. K., Ohdar, R. K., \& Mahapatra, S. S. (2010). Parametric appraisal of mechanical property of fused deposition modelling processed parts. Materials \& Design, 31(1), 287-295.

Sugavaneswaran, M., \& Arumaikkannu, G. (2018). Additive manufactured multi-material structure with directional specific mechanical properties based upon classical lamination theory. Rapid Prototyping Journal, 24, 1212-1220.

Xia, Y., Xu, K., Zheng, G., Zou, R., Li, B., \& Hu, P. (2019). Investigation on the elasto-plastic constitutive equation of parts fabricated by fused deposition modeling. Rapid Prototyping Journal, 25, 592-601.

Zhou, Y. G., Su, B., \& Turng, L. S. (2017). Deposition-induced effects of isotactic polypropylene and polycarbonate composites during fused deposition modeling. Rapid Prototyping Journal, 23, 869-880.

Zhou, Y. G., Zou, J. R., Wu, H. H., \& Xu, B. P. (2020). Balance between bonding and deposition during fused deposition modeling of polycarbonate and acrylonitrile-butadiene-styrene composites. Polymer Composites, 41(1), 60-72.

Zou, R., Xia, Y., Liu, S., Hu, P., Hou, W., Hu, Q., \& Shan, C. (2016). Isotropic and anisotropic elasticity and yielding of 3D printed material. Composites Part B: Engineering, 99, 506-513.

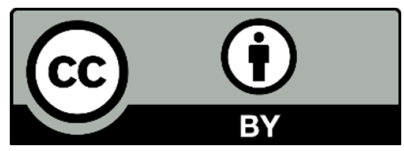

(C) 2022 by the authors; licensee Growing Science, Canada. This is an open access article distributed under the terms and conditions of the Creative Commons Attribution (CC-BY) license (http://creativecommons.org/licenses/by/4.0/). 